\title{
Experimental Studies of Scaling Behavior of a Quantum Hall System with a Tunable Landau Level Mixing
}

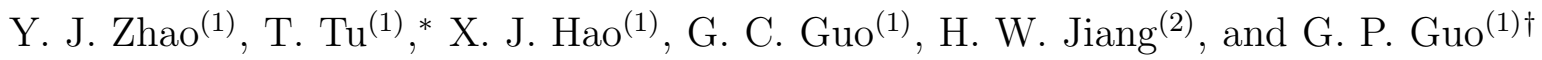 \\ (1) Key Laboratory of Quantum Information, \\ University of Science and Technology of China, \\ Chinese Academy of Sciences, Hefei 230026, P. R. China \\ (2) Department of Physics and Astronomy, \\ University of California at Los Angeles, \\ 405 Hilgard Avenue, Los Angeles, CA 90095, USA
}

(Dated: November 1, 2018)

\begin{abstract}
Temperature dependence of the longitudinal and Hall resistance is studied in the regime of localization-delocalization transition. We carry out measurements of a scaling exponent $\kappa$ in the Landau level mixing region at several filling factors. The localization exponent $\gamma$ is extracted using an approach based on the variable range hopping theory. The values of $\gamma$ and $\kappa$ are found to be universal, independent of filling factor in our sample. We can conclude that although Landau level mixing can change the degeneracy of a quantum Hall state, the value of the scaling exponent remains the same for a given sample that contains a fixed disorder profile.

PACS numbers: 73.43.Nq, 71.30.+h, 72.20.My
\end{abstract}

*Electronic address: tutao@ustc.edu.cn

†Electronic address: gpguo@ustc.edu.cn 
The localization problem has been studied intensively in the past decades $[1,2]$. It's well accepted that there is no true delocalization state in non-interaction two-dimensional electron gas (2DEG) provided with disorder. However when a strong external magnetic field is applied, a delocalized state is formed at the center of the Landau level, while the electronic state is localized away from this discrete energies. The coexistence of localized and delocalized states is essential for the quantum Hall effect. Phase transitions between localized and delocalized states will occur when the Fermi level is swept from one Landau level to another. The relative position of the Fermi level and Landau levels can be experimentally changed by varying the external magnetic field and/or the carrier density. Since this phase transition is considered as a continuous quantum phase transition, finite size scaling theory [3] can be applied in the critical regime, where the resistance tensor scales as $R_{u v}=R_{c} f(L / \xi)$ for a sample of finite size L. Here $f$ is a scaling function which can be derived from the microscopic calculation. The localization length $\xi$ diverges as the Fermi level approaches the center of a Landau level $E_{c}$ as $\xi \propto\left|E-E_{c}\right|^{-\gamma}$ with an exponent $\gamma$. The interaction induced quantum phase coherence length sets the effective sample size $L$ and its temperature dependence of $L \propto T^{-\frac{p}{2}}$. Then one obtains $R_{u v}=R_{c} f\left(\left|B-B_{c}\right| T^{-\kappa}\right)$, the scaling function of both the longitudinal resistance $R_{x x}$ and the Hall resistance $R_{x y}$, where the scaling exponent is expressed as $\kappa=p / 2 \gamma$. Approaching zero temperature, the maximum slope in the Hall resistance $R_{x y}$ with varying magnetic field $B$ diverges as a power law $\left.\frac{d R_{x y}}{d B}\right|_{B_{c}} \propto T^{-\kappa}$, while the half width for the longitudinal resistance $R_{x x}$ vanishes as $\Delta B \propto T^{\kappa}$ [4].

Despite the intense studies of the scaling behaviors of the quantum Hall systems $[4,5,6,7,8,9,10,11,12]$, several issues are still unsettled. For example, when the electron spin is unresolved (i.e., the two spin states of the Landau levels are mixed), the interaction of electrons in different Landau levels complicate the problem and draw into question whether this universality of scaling behavior can be preserved. Experimentally, there was an indication that the exponent $\kappa$ can be changed by a factor of two when the system becomes spin-degenerate $[13,14]$. On the other hand, theoretical works $[15,16]$, based on two different models, concluded that Landau level mixing will not change the universality of this phase transition. The earlier experimental studies on the localization-delocalization phase transition in the Landau level mixing regime is focused on the spin unresolved plateau transitions $[13,14]$, which involves the adjacent Landau levels with spin degeneracy. The experiment presented in this paper is carried out in a two subband system in which two 
Landau levels (LLs) with different subband, Landau level index, or spin state can be controllably mixed either by varying electron density or by changing magnetic field. The main objective of the study is to determine experimentally the contribution of the Landau level mixing on the scaling behavior.

The sample we investigated is grown by molecular-beam epitaxy and consists of a symmetrical modulation-doped $24 \mathrm{~nm}$ wide single GaAs quantum well bounded on each side by Si $\delta$-doped layers of AlGaAs. Heavy doping creates a very dense 2DEG, resulting in the filling of two subbands in the well when at low temperature. As determined from the Hall resistance data and Shubnikov-de Haas oscillations in the longitudinal resistance, the total density is $n=8.0 \times 10^{11} \mathrm{~cm}^{-2}$, where the first and the second subband have a density of $n_{1}=6.1 \times 10^{11} \mathrm{~cm}^{-2}$ and $n_{2}=1.9 \times 10^{11} \mathrm{~cm}^{-2}$. The sample has a low-temperature mobility $\mu=4.1 \times 10^{5} \mathrm{~cm}^{2} / \mathrm{V} \mathrm{s}$, which is extremely high for a 2 DEG with two filled subbands. The samples are patterned into Hall bars using standard lithography techniques. A NiCr top gate is evaporated on the top of the sample, approximately $350 \mathrm{~nm}$ away from the center of the quantum well. By applying a negative gate voltage on the $\mathrm{NiCr}$ top gate, the electron density can be tuned continuously. Magneto-transport measurements were carried out in an Oxford Top-Loading Dilution Refrigerator with a base temperature of $15 \mathrm{mK}$. To measure the longitudinal and Hall resistance $R_{x x}$ and $R_{x y}$, we used a standard ac lock-in technique with electric current ranging from $10 \mathrm{nA}$ to $100 \mathrm{nA}$ at a frequency of $11.3 \mathrm{~Hz}$.

At first we measure the phase diagram of the two-subband sample, which is the gray-scale plot of longitudinal resistance $R_{x x}$ as a function of magnetic field $B$ and electron density $n$, as shown in Fig.1. In this gray-scale map, bright lines represent peaks in $R_{x x}$, where the electron state is delocalized; while the dark regions are the minimums, corresponding to the quantum Hall states, where localized state occurs. From this diagram we can find the spin is resolved when magnetic field is greater than 2.2 Tesla. The ringlike structures at even filling factor is due to interaction between the two set of Landau levels [17]. This situation occurs when two Landau levels from different subband are brought into degeneracy or mixing by tuning electron density and magnetic field. According to the standard Landau level fan diagram as illustrated schematically in Fig.1 inset, point 6A represents the mixing of Landau level $(A, 0, \uparrow)$ and $(S, 2, \downarrow)$, while point $6 \mathrm{~B}$ corresponds to mixing of $(A, 0, \downarrow)$ and $(S, 2, \uparrow)$. Here we label the single-particle levels $(i, N, \sigma)$, and $i(=S, A), N$, and $\sigma(=\uparrow, \downarrow)$ are the subband (symmetry or antisymmetry), orbital and spin quantum numbers. Since 


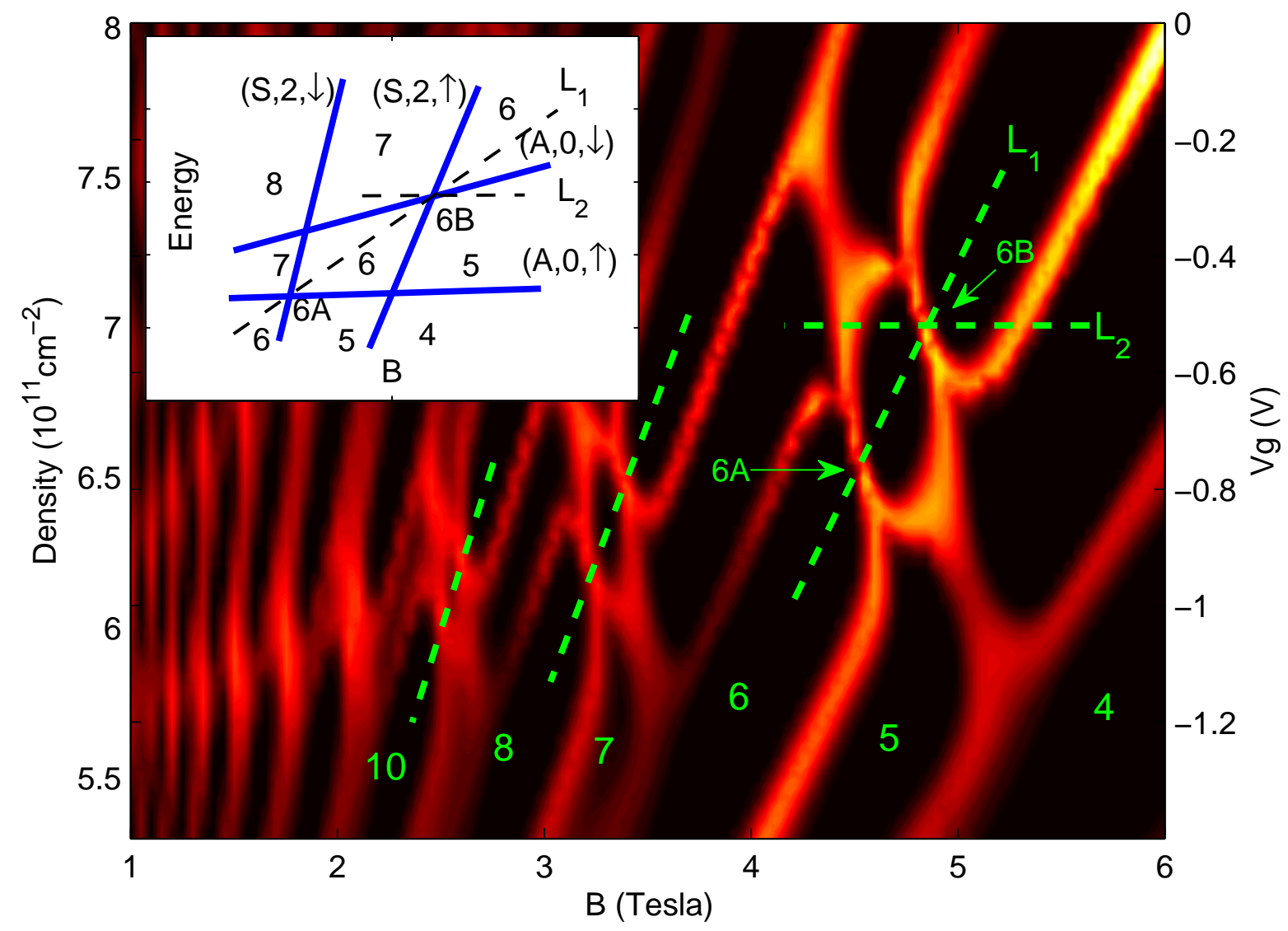

FIG. 1: (color online). The phase diagram of $R_{x x}$ against gate voltage $V_{g}$ and magnetic field $B$. Numbers in the graph label the regions with corresponding filling factor. The horizontal dashed line $L_{2}$ is the conventional measurement trace of plateau transition between filling factor 5-6-7, while the three tilted dashed lines are measurement path at filling factor 6, 8, 10 separately. Especially at the region of filling factor $\nu=6,6 \mathrm{~A}$ and $6 \mathrm{~B}$ indicate the Landau level mixing points where the $R_{x x}$ peaks occur. Inset is the Landau level diagram at $\nu=6$.

Landau level mixing influence the pattern of phase diagram profoundly, it's interesting to find out whether this mixing will change the universality of scaling behavior of localizationdelocalization transition or not.

By changing the magnetic field whistle varying the gate voltage, we can guide the measurement trace crossing bright lines in the phase diagram and then undergoing localizeddelocalized transitions. Dashed lines in Fig.1 illustrate such measurement traces. It should emphasis that the tilted traces crossing ringlike structures at filling factor $\nu=6,8,10$, are 


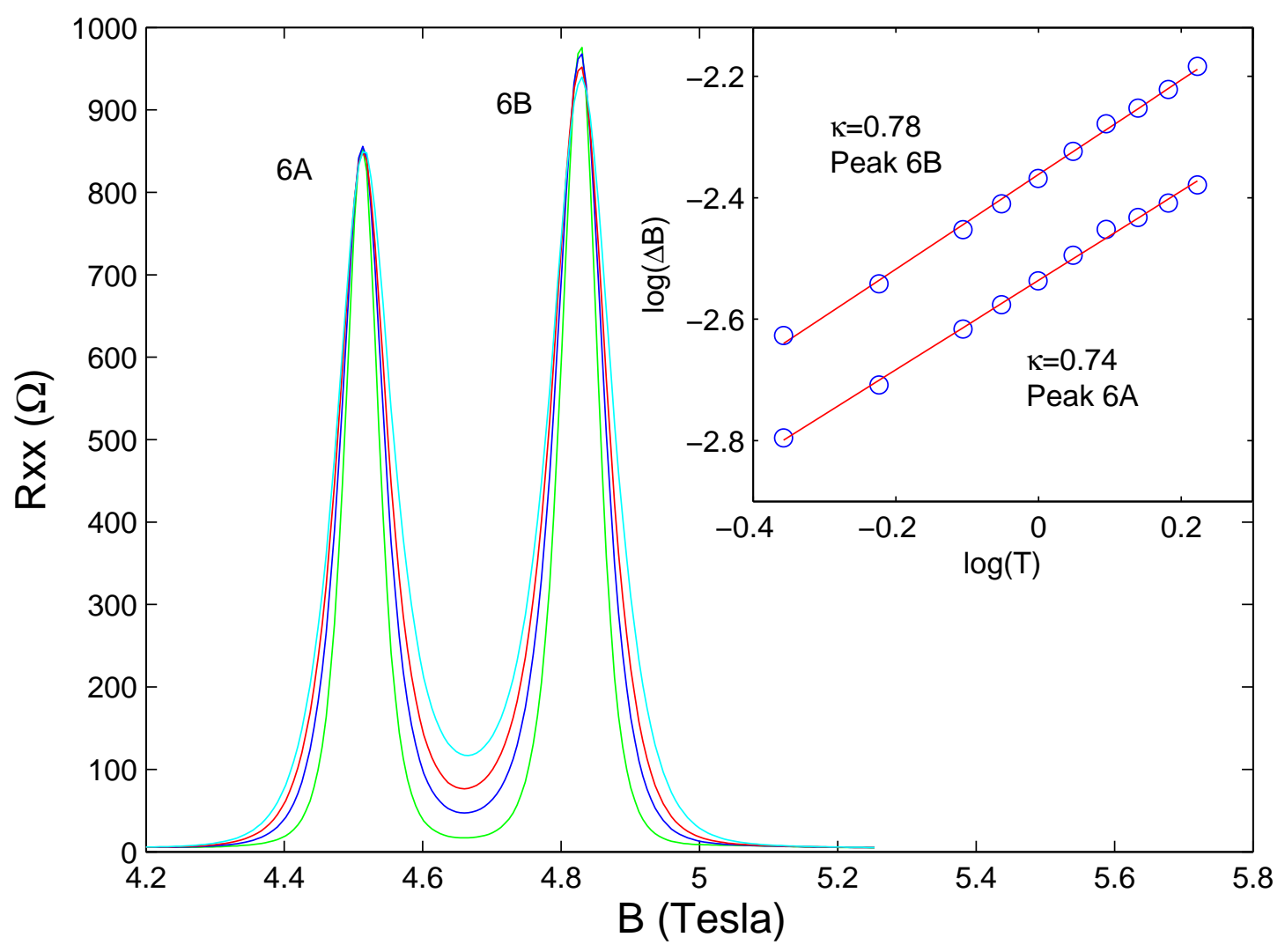

FIG. 2: (color online). The longitudinal resistance $R_{x x}$ at different temperatures along trace $L_{1}$. The insets shows the half-width $\Delta B$ vs temperature $T$ corresponding to the two peaks in the $R_{x x}$ curve.

important for our study since they cross the Landau level mixing points exactly. Fig. 2 shows the temperature dependence measurement of longitudinal resistance $R_{x x}$ following trace $L_{1}$ across the Landau level mixing region at filling factor $\nu=6$. The two peaks which result from intersection of trace $L_{1}$ and the ringlike structure, are noted as $6 \mathrm{~A}$ and $6 \mathrm{~B}$, and each shows a broaden as the temperature increasing from $50 \mathrm{mK}$ to $1.2 \mathrm{~K}$. In order to compare the experiment result with conventional plateau-plateau transition situation, we also measured the transition between filing factor $\nu=5,6,7$ along trace $L_{2}$. The corresponding result is plotted in Fig.3.

Temperature scaling exponent $\kappa$ can be extracted from power-law fit for the half width $\Delta B \propto T^{\kappa}$, which yield values of 0.74 and 0.78 for peak $6 \mathrm{~A}$ and $6 \mathrm{~B}$ respectively (Fig. 2 inset). It should be noticed that, along line $L_{1}$ the Hall resistance keeps constant since the 


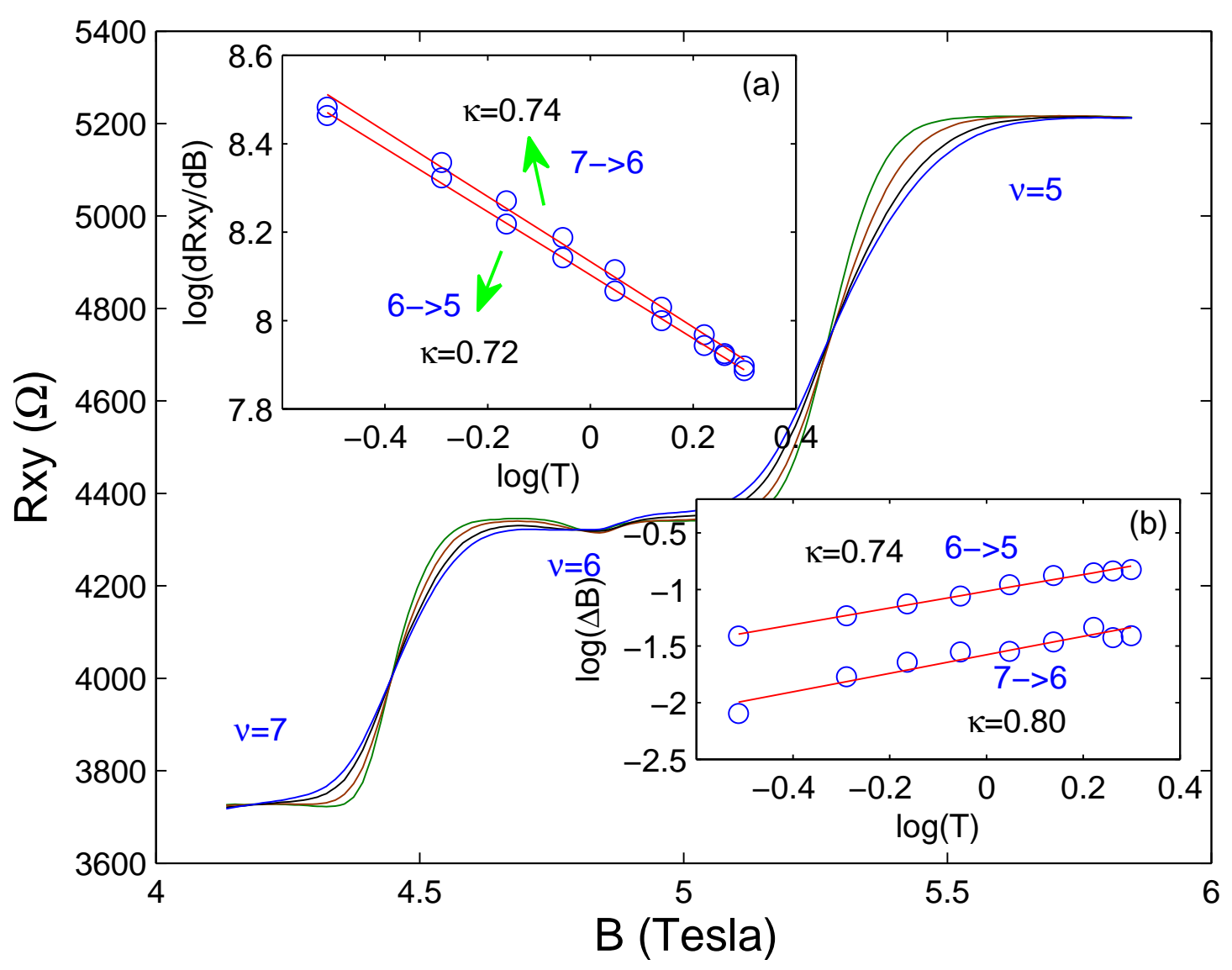

FIG. 3: (color online). The Hall resistance $R_{x y}$ at different temperatures along line $L_{2}$. Inset (a) shows the scaling exponent $\kappa$ extracted from the temperature dependence of $R_{x y}$ slop, while inset (b) gives $\kappa$ derived from scaling of half width of $R_{x x}$ peak.

the measurement trace will not change the filling factor. On the other hand, as shown in Fig.3 inset, the scaling exponent is extracted from half width $\Delta B$ as well as maxima of $\frac{d R_{x y}}{d B}$, which shows $\kappa$ ranges within $\kappa=0.75 \pm 0.05$. The result is consistent with what occurs at Landau level mixing region within experiment error. The scaling in the Landau level mixing region at other filling factor $(\nu=8,10)$ are also examined. All the results fall into range $\kappa=0.75 \pm 0.05$. Our experiment results turn out that the exponent $\kappa$ is universal in respect to the filling factor within the experimental error, whatever there exists Landau level mixing or not.

Additionally, an alternative approach to scaling can be used for a direct evaluation of the localization length $\xi$. Polyakov and Shklovskii argued that the mechanism for the con- 
ductivity peak broadening is variable range hopping (VRH) in the presence of Coulomb interactions[18]. The scaling function of VRH conductivity is given as

$$
\sigma_{x x}=\sigma_{0} \exp \left(-\sqrt{\frac{T_{0}}{T}}\right), T_{0}(B) \propto \frac{e^{2}}{\varepsilon \xi(B)} .
$$

The characteristic temperature $T_{0}$ is determined by the coulomb interaction at length scale given by localization length $\xi$. Here $\sigma_{0} \propto 1 / T$ is a temperature dependent parameter. As mentioned above, the localization length $\xi(B)$ diverges as $\xi(B) \propto\left|B-B_{c}\right|^{\gamma}$, combine this relations yields

$$
\sigma_{x x}=\sigma^{*} s \exp \left(-\sqrt{T^{*} s}\right), s=\frac{\left|B-B_{c}\right|^{\gamma}}{T} .
$$

Where $s$ is the scaling variable, $\sigma^{*}$ and $T^{*}$ are constant. Using this formula between conductivity $\sigma$ and $\xi$, we can easily and directly obtain the localization length $\xi$ and corresponding localization exponent $\gamma$.

We fit the experiment data using the scaling function Eq.2 as shown in Fig.4. By varying the localization length exponent $\gamma$ we can make all the data in the phase transition region along line $L_{1}$ fall into a single line. Therefore we find the fit is perfect, and we get $\gamma \approx 1.3$. This result is quite different from that of previous experimental $[6,9,10]$ and theoretical works $[19,20,21]$ which give a value $\gamma \approx 2.3$. We also investigate the localization length exponent at other region of the phase diagram, including Landau level mixing points at higher filling factor $(\nu=8,10)$, and conventional plateau-plateau transition situation along line $L_{2}$ (between plateau $\nu=5,6,7$ ). Within experimental error, the localization length exponent $\gamma$ is found to be universal, independent of filling factor, Landau level mixing situation. All the cases give a perfect fit of $\gamma=1.3 \pm 0.2$.

The scaling exponent $\kappa$ and localization length exponent $\gamma$ both show a deviation from conventional values. Even these deviations, however, we should emphasis that the universality of scaling behavior exist for our sample, with exponent values of $\kappa=0.75 \pm 0.05$ and $\gamma=1.3 \pm 0.2$. We can conclude that although Landau level mixing can change the degeneracy of a quantum Hall state, this mixing will not change the scaling behavior of our sample. Since the two exponents are extracted independently, we think they reveal more valuable information beneath the scaling phenomenon. Using the relation $\kappa=p / 2 \gamma$, we can get inelastic scattering rate exponent $p \approx 2$, which is exactly the zero-field clean limit for 


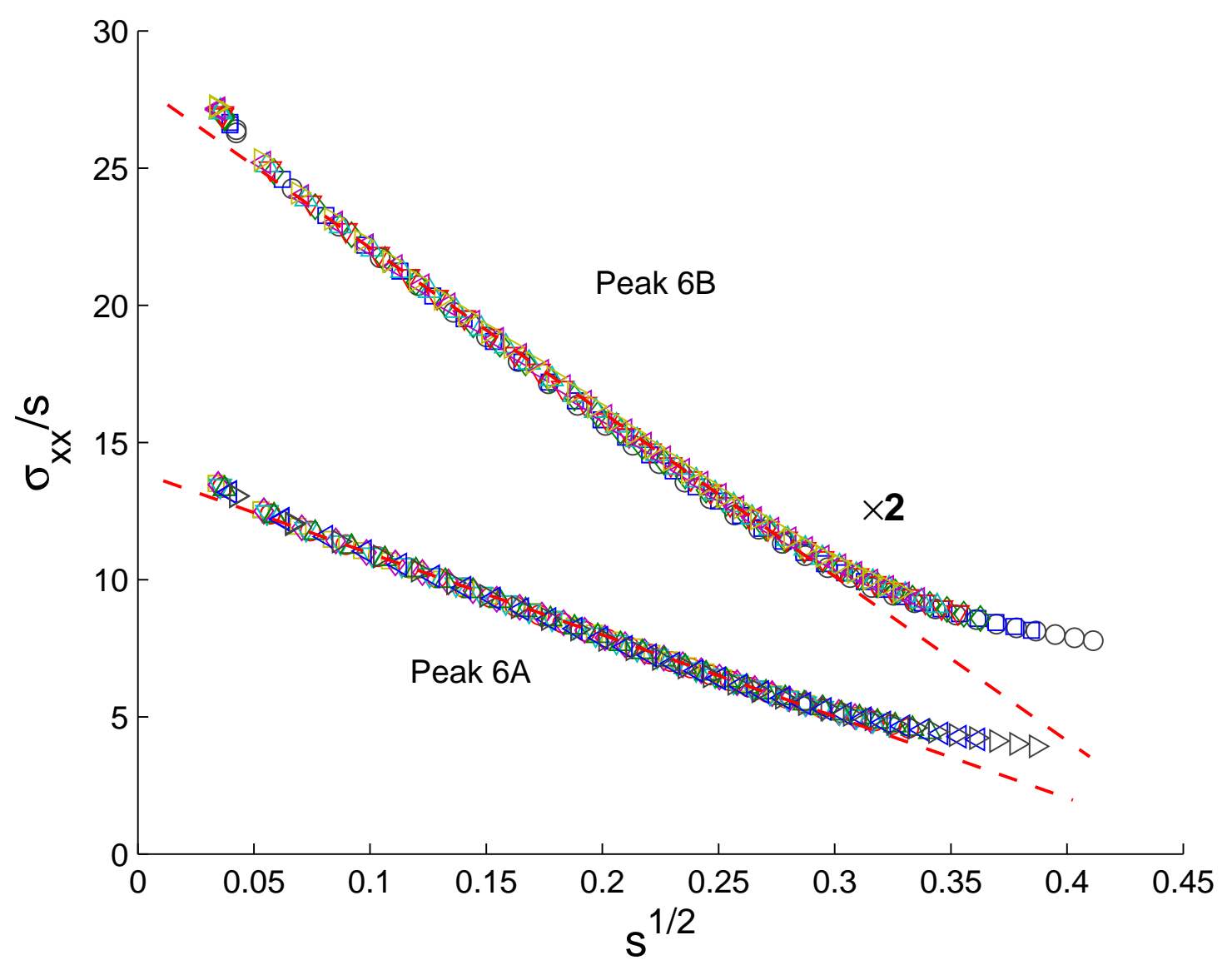

FIG. 4: (color online). Longitudinal conductivity $\sigma_{x x}$ as a function of the scaling variable $s$ in the transition regime at $\nu=6$ along line $L_{1}$. The dashed line is indicated for fitting to Eq.(2).

electron-electron scattering in two-dimensional Fermi liquids [21, 22, 23]. This result is also consistent with most of the experimental works $[8,11]$.

For the samples we investigated, the deviation of $\kappa$ from the conventional value $\kappa \approx 0.42$ can be attributed to the effect from quantum localization toward classical percolation (using the value of $p$ and the $\kappa=p / 2 \gamma$ relationship, one can obtain $\kappa \approx 0.42$ and $\kappa \approx 0.75$ corresponding to $\gamma \approx 2.4$ and $\gamma \approx 1.3$ respectively). Most of direct measurements of localization length exponent $\gamma$ yield a universal value of 2.4, which is predicted by theoretical calculations based on a network model of quantum percolation [19, 20, 21]. However, our direct measurement of $\gamma$ through $\mathrm{VRH}$ method yields the value of 1.3, which coincides with $\gamma=4 / 3$, a value obtained with theories of classical percolation [21, 24]. It shows that classical percolation dominates scaling behavior in our samples. Theoretical work[25] suggest 
that in presence of long range potential fluctuation due to the remote ionized impurities in AlGaAs, a larger crossover region will occur where classical percolation applies. On the other hand, in [12] the author provided a systematic investigation on the influence of type of disorder potential on the scaling behavior. The result turned out that only for short range alloy potential fluctuations the scaling exponent is consistent with the conventional value $\kappa \approx 0.42$. However for the long range potential fluctuation situation, the exponent increase towards the classical value of 0.75 . We think our results are coincide with previous observations. This suggests that whatever there exists Landau level mixing or not, the value of the scaling exponent remains the same for a given sample that contains a fixed disorder profile.

There is also a different approach for the explanation of this phenomena. Avishai and Meir [26] studied critical exponent $\gamma$ in the presence of spin-orbit scattering. Their numerical calculations show that the exponent is very close to $\gamma=4 / 3$ and consistent with our results. The spin-orbit interaction is also investigated in GaAs/AlGaAs quantum well recently [27], which suggested the this interaction arises from the structural inversion asymmetry of the heterostructure, and hence is influenced significantly by applying gate voltage. Since our sample utilizing a front gate to tune the carrier density, it is possible for the enhancement of spin-orbit interaction, which eventually induces localization length exponent $\gamma \approx 1.3$.

In conclusion, temperature dependence of the Hall and longitudinal resistance of twosubband quantum Hall samples is measured in our experiments. The emphasis is focused on the scaling behavior of the localized-delocalized transition in the Landau level mixing region at several filling factors (from 5 to 11). The scaling exponent $\kappa$ is extracted by power law fit of half width of $R_{x x}$ peak dependence on temperature. Additionally we extract quantitative information of the localization exponent $\gamma$ through an approach based on the variable range hopping theory. The main result is that we find an accurate universality for both the scaling exponent $\kappa \approx 0.75$ and the localization length exponent $\gamma \approx 1.3$ within the experimental error. In comparison with results obtained from conventional plateau-plateau transition, it turns out although Landau level mixing can change the degeneracy of a quantum Hall state, the value of the scaling exponent remains the same for a given sample that contains a fixed disorder profile. Also several explanations are supposed to explain the value of $\gamma$ and $\kappa$ 's deviation from the conventional values.

This work was funded by National Fundamental Research Program, the Innovation funds 
from Chinese Academy of Sciences, NCET-04-0587, and National Natural Science Foundation of China (Grant No. 60121503, 10574126, 10604052).

[1] B. Huckestein, Rev. Mod. Phys. 67357 (1995).

[2] S. L. Sondhi, S. M. Girvin, J. P. Carini and D. Shahar, Rev. Mod. Phys. 69, 315 (1997).

[3] A. M. Pruisken, Phys. Rev. Lett. 61, 1297 (1988).

[4] H. P. Wei, D. C. Tsui, M. Paalanen, and A. M. Pruisken, Phys. Rev. Lett. 61, 1294 (1988).

[5] S. Koch, R. J. Haug, K. v. Klitzing and K. Ploog, Phys. Rev. B 43, 6828 (1991).

[6] S. Koch, R. J. Haug, K. v. Klitzing and K. Ploog, Phys. Rev. Lett. 67, 883 (1991).

[7] H. P. Wei, S. Y. Lin and D. C. Tsui, Phys. Rev. B 45, 3926 (1992).

[8] L. W. Engel, D. Shahar, C. Kurdak, and D. C. Tsui, Phys. Rev. Lett. 71, 2638 (1991).

[9] F. Hohls, U. Zeitler and R. J. Haug, Phys. Rev. Lett. 86, 5124 (2001).

[10] F. Hohls, U. Zeitler and R. J. Haug, Phys. Rev. Lett. 88, 036802 (2002).

[11] F. Hohls, U. Zeitler, R. J. Haug, R. Meisels, K. Dybko and F. Kuchar, Phys. Rev. Lett. 89, $276801(2002)$.

[12] W. Li, G. A. Csathy, D. C. Tsui, L. N. Pfeiffer, and K. W. West, Phys. Rev. Lett. 94, 206807 (2005).

[13] H. P. Wei, S. W. Hwang, D. C. Tsui and A. M. Pruisken, Surf. Sci. 229, 34 (1990).

[14] S. W. Hwang, H. P. Wei, L. W. Engel, D. C. Tsui, and A. M. Pruisken, Phys. Rev. B 48, 11416 (1993).

[15] Z. Wang, D.-H. Lee and X.-G. Wen, Phys. Rev. Lett. 72, 2454 (1994).

[16] D. K. Lee and J. T. Chalker, Phys. Rev. Lett. 72, 1510 (1994).

[17] X. C. Zhang, D. R. Faulhaber and H. W. Jiang, Phys. Rev. Lett. 95, 216801 (2005).

[18] D. G. Polyakov and B. I. Shklovskii, Phys. Rev. Lett. 70, 3796 (1993).

[19] J. T. Chalker and P. D. Coddington, J. Phys. C 21, 2665 (1988).

[20] B. Huckestein and B. Kramer, Phys. Rev. Lett. 64, 1437 (1990).

[21] D.-H. Lee, Z. Wang and S. Kivelson, Phys. Rev. Lett. 70, 4130 (1993).

[22] D.-H. Lee and Z. Wang, Phys. Rev. Lett. 76, 4014 (1996).

[23] B. Huckestein and M. Backhaus, Phys. Rev. Lett. 82, 5100 (1999).

[24] S. A. Trugman, Phys. Rev. B 27, 7539 (1983). 
[25] J. E. Moore, Phys. Rev. B 65, 241309 (2002).

[26] Y. Avishai and Y. Meir, Phys. Rev. Lett. 89, 076602 (2002).

[27] K. Hashimoto, K. Muraki, N. Kumada, T. Saku and Y. Hirayama, Phys. Rev. Lett. 94, 146601 (2005). 